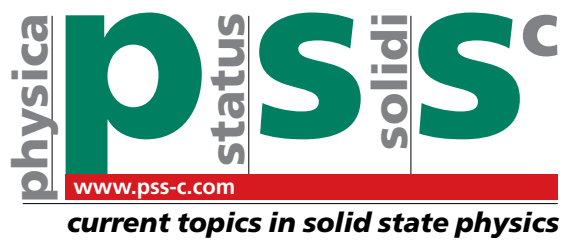

\title{
Resonant energy transfer in electron-driven proton pumps
}

\author{
A. Yu. Smirnov ${ }^{1,2,3}$, L. G. Mourokh ${ }^{*, 1,3,4,5}$, and Franco Nori ${ }^{1,6}$ \\ ${ }^{1}$ Frontier Research System, The Institute of Physical and Chemical Research (RIKEN), Wako-shi, Saitama 351-0198, Japan \\ ${ }^{2}$ CREST, Japan Science and Technology Agency, Kawaguchi, Saitama 332-0012, Japan \\ ${ }^{3}$ Quantum Cat. Analytics, 175167 St. \#E11, Brooklyn, New York 11204, USA \\ ${ }^{4}$ Department of Physics, Queens College, The City University of New York, Flushing, New York 11367, USA \\ ${ }^{5}$ Department of Engineering Science and Physics, College of Staten Island, The City University of New York, Staten Island, \\ New York 10314, USA \\ ${ }^{6}$ Center for Theoretical Physics, Physics Department, University of Michigan, Ann Arbor, MI 48109, USA
}

Received 9 July 2007, revised 6 August 2007, accepted 25 August 2007

Published online 23 October 2007

PACS 05.60.Gg, 73.63.-b, 87.15.Aa, 87.16.Uv

* Corresponding author: e-mail levmurokh@physics.qc.edu, Phone: +1 718997 3363, Fax: +1 7189973349

We examine proton and electron transport in living cell membranes and show that the Coulomb interaction leads to a resonant energy transfer, from the electrons to protons during their simultaneous tunnelings, which allows the proton pump to work. The proton pump efficiency is controlled by the electron voltage build-up, external temperature, and the molecular electrostatics driving the electron and proton energies to their resonant conditions. We also show that physiological (e.g., human body) temperatures are optimal for the operation of this proton pump.
1 Introduction Charge and energy transfers in living cells require nanoscale machinery working with high efficiency and reliability. An example of such nanomachine is the cytochrome $c$ oxidase $(\mathrm{CcO})$ which converts atmospheric oxygen into water, reducing the energy of electrons (reduction/oxidation, or redox, process) and simultaneously creating a proton gradient across the inner mitochondrial membrane (proton pump) [1]. This membrane separates regions with different proton concentrations, and the proton gradient is used for driving the biological motor of adenosine triphosphate (ATP) synthase, which converts mechanical motion into the chemical energy stored in ATP molecules. In recent years, the structure of $\mathrm{CcO}$ has been determined for various organisms, but the specific mechanism of the proton pump remains elusive [2].

Recent progress in nanotechnology brings the parameters of modern electronic devices closer to the scales of living cell machinery. This makes it possible both to mimic biological systems for device purposes and to analyze the electron and proton transport mechanisms in living cells using methods of nanoelectronics and nanomechanics. Here, we apply such methods to examine the electroni- cally-controlled proton transfer through an inner mitochondrial membrane and to try to understand the elusive mechanism of proton pumps. To create and maintain the proton gradient, it is necessary to transfer energy from the intra-membrane electron transport chain to the proton subsystem. We propose a model for this energy transduction based on the Förster-type energy exchange [3], i.e. when the simultaneous electron and proton tunnelings are accompanied by a transfer of energy under resonant conditions between the electrons and protons. It should be emphasized that such quantum-mechanical processes [4] (i.e. electron and proton tunnelings) are very important for living cell dynamics, despite temperatures much larger than that of conventional nanoelectronics. We show below that our proton pump mechanism operates optimally at human body temperatures (about $36^{\circ} \mathrm{C}$ ).

2 Formulation We examine the situation where the electron transport between the left, $L$, and right, $R$, sites embedded into the inner membrane of the mitochondrion is accompanied by the proton transfer from the "lower" $(D)$ protonable site that is close to the negative (inner) side of 
the membrane to the "upper" $(U)$ protonable site located nearby the positive (outer) surface of the membrane (Fig. $1)$.

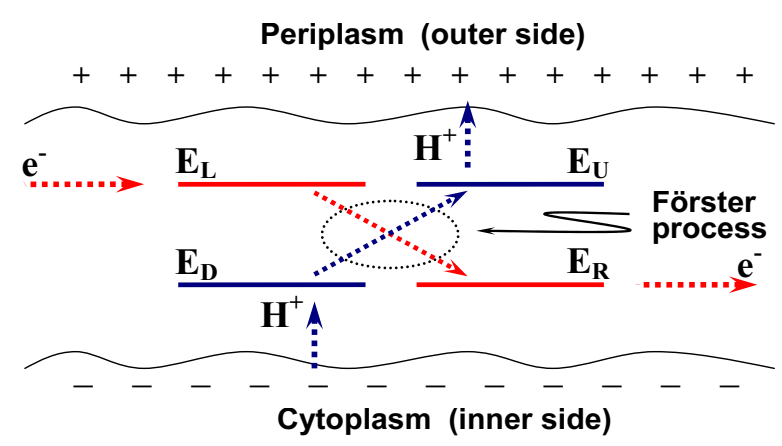

Figure 1 Schematic diagram of the Förster-type energy exchange between electron and proton subsystems. The electron transfer from left to right is accompanied by the proton transfer from the site $D$ with lower energy to the site $U$ with higher energy.

In the case of $\mathrm{CcO}$, a specific site (known as heme a) and a binuclear site can be considered $[5,6]$ as a double-dot system for electrons with two dots (sites) having different energies $E_{L}$ and $E_{R}$ [6]. For the proton component, the two proton dots (sites) having different energies, $E_{D}$ and $E_{U}$, correspond to [6] a carboxilyc residue E278 from the negative (inner) side of the membrane and an protonable site from the positive (outer) side of the membrane. It is assumed [6] that the left (right) electron site is coupled only to the left (right) electronic reservoir and the proton $D$ and $U$ sites are also coupled only to the corresponding proton reservoirs. The electron and proton tunnelings between their two sites are described by the tunneling splittings, $\Delta_{a}$ and $\Delta_{b}$, respectively. In addition, the Coulomb interaction between electrons and protons localized on the corresponding sites leads to the Förster transfer [3], i.e., to the process when the energy is transferred from the electrons to protons (or vice versa) during their simultaneous tunnelings. The same mechanism is responsible for the fluorescence resonant energy transfer (FRET) in biological systems [7] as well as for exciton transfer in condensed matter [8]. To be effective, the Förster transfer requires a resonant condition: when the energy difference between the initial $(L)$ and final $(R)$ electron states is equal to the energy separation of the final $(U)$ and the initial $(D)$ proton states: $E_{L}-E_{R} \approx E_{U}-E_{D}$. We show that, in the presence of the electron-proton Förster interaction, the voltage applied between the left and the right electron reservoirs (redox potential) can be used for generating a proton current in the direction of higher proton concentration, thus realizing a proton pump.

To analyze this process quantitatively, we examine an electron double-dot connected to the left and right electron reservoirs and a proton double-dot connected to the "up" and "down" proton reservoirs. The Hamiltonian describing double-dots is given by

$H=\sum E_{\alpha} a_{\alpha}^{+} a_{\alpha}+\sum E_{\beta} b_{\beta}^{+} b_{\beta}-\Delta_{a} a_{L}^{+} a_{R}-\Delta_{a}^{*} a_{R}^{+} a_{L}$

$-\Delta_{b} b_{D}^{+} b_{U}-\Delta_{b}^{*} b_{U}^{+} b_{D}-\lambda a_{L}^{+} a_{R} b_{D}^{+} b_{U}-\lambda^{*} a_{R}^{+} a_{L} b_{U}^{+} b_{D}$

and the coupling of the double dots to the leads has the form

$$
H_{T}=-\sum t_{k \alpha} c_{k \alpha}^{+} a_{\alpha}-\sum T_{k \beta} d_{k \beta}^{+} b_{\beta}+H . c .
$$

Here, $a_{\alpha}^{+}\left(a_{\alpha}\right)$ and $b_{\beta}^{+}\left(b_{\beta}\right)$ are the creation (annihilation) operators in the $\alpha$-sites for the electrons and in the $\beta$-sites for the protons, respectively ( $\alpha=L ; R ; \beta=D ; U) ; E_{\alpha}$ and $E_{\beta}$ are the energies of electrons and protons in the corresponding $\alpha$ - and $\beta$-dot, respectively. Terms with tunneling amplitudes $\Delta_{a}$ and $\Delta_{b}$ describe the conventional electron and proton tunnelings, whereas the resonant Förster double-tunneling is described by the terms with the coupling constant. The direct Coulomb coupling of electrons and protons only leads to the renormalization of their energies; however, the overlap of their wave function produces the Coulomb terms responsible for the Förster-type interaction $[3,9]$.

Evaluating the equations of motion for electron and proton operators, we obtain self-consistent set of equations for electron and proton populations in the form:

$$
\begin{aligned}
& \left\langle n_{L}\right\rangle=\int \frac{d \omega}{2 \pi}\left(\gamma_{L} f_{L}(\omega)\left|F_{L}(\omega)\right|^{2}+\left|\Delta_{a}\right|^{2} \gamma_{R}\left|G_{L}(\omega)\right|^{2}\left|G_{R}(\omega)\right|^{2}\right) \\
& +|\lambda|^{2}\left\langle N_{U}\right\rangle\left(1-N_{D}\right) \gamma_{R} \int \frac{d \omega}{2 \pi} f_{R}\left(\omega-\frac{E_{U}-E_{D}}{2}\right) \\
& \times\left|G_{L}\left(\omega-\frac{E_{U}-E_{D}}{2}\right)\right|^{2}\left|G_{R}\left(\omega-\frac{E_{U}-E_{D}}{2}\right)\right|^{2}
\end{aligned}
$$

$<n_{R}>$ is obtained from Eq. (3) by replacing $L$ by $R, D$ by $U$, and vice versa. Essentially identical equations hold for the proton populations, $\left\langle N_{U}\right\rangle$ and $\left\langle N_{D}\right\rangle$. The Green's functions involved in Eq. (3) (as well as in the equations for the proton populations, with replacement of $\alpha$ by $\beta$ and $\gamma$ by $\Gamma)$ are given by

$$
G_{\alpha}(\omega)=\frac{1}{\omega-E_{\alpha}+i \gamma_{\alpha} / 2}
$$

and

$$
F_{\alpha}(\omega)=\frac{1}{\omega-E_{\alpha}+i \gamma_{\alpha} / 2-\Sigma_{\alpha}(\omega)},
$$

where $\gamma_{\alpha}\left(\Gamma_{\beta}\right)$ are the electron (proton) level broadenings due to the couplings to their respective leads, $f_{\alpha}\left(f_{\beta}\right)$ are the Fermi distribution functions in the corresponding electron (proton) reservoirs with the chemical potential $\mu_{L^{-}} \mu_{R}=V_{e}$ $\left(\mu_{U}-\mu_{D}=V_{p}\right)$, and $V_{e}\left(V_{p}\right)$ are the voltages build-up in the electron (proton) subsystems, respectively. The self-energy 
$\Sigma_{L}(\omega)$ is given by

$\Sigma_{L}(\omega)=\left|\Delta_{a}\right|^{2} G_{R}(\omega)$

$+|\lambda|^{2}\left\langle N_{D}\right\rangle\left(1-\left\langle N_{U}\right\rangle\right) G_{R}\left(\omega+E_{D}-E_{U}\right)$

with $\Sigma_{R}(\omega), \Sigma_{U}(\omega)$, and $\Sigma_{D}(\omega)$ having similar forms.

Using the equations of motion for electron and proton operators of the leads, we obtain expressions for the electron and proton currents in terms of the steady-state populations, Fermi distributions, and Green's functions, as

$I_{e}=\left|\Delta_{a}\right|^{2} \gamma_{L} \gamma_{R} \int \frac{d \omega}{2 \pi}\left|G_{L}(\omega)\right|^{2}\left|G_{R}(\omega)\right|^{2}\left(f_{L}(\omega)-f_{R}(\omega)\right)$

$+|\lambda|^{2} \gamma_{L} \gamma_{R} \int \frac{d \omega}{2 \pi}\left|G_{L}\left(\omega+\frac{E_{U}-E_{D}}{2}\right)\right|^{2}\left|G_{R}\left(\omega-\frac{E_{U}-E_{D}}{2}\right)\right|^{2}$

$\times\left[\left\langle N_{D}\right\rangle\left(1-\left\langle N_{U}\right\rangle\right) f_{L}\left(\omega+\frac{E_{U}-E_{D}}{2}\right)\right.$

$\left.-\left\langle N_{U}\right\rangle\left(1-\left\langle N_{D}\right\rangle\right) f_{R}\left(\omega-\frac{E_{U}-E_{D}}{2}\right)\right]$

and

$I_{p}=\left|\Delta_{b}\right|^{2} \Gamma_{D} \Gamma_{U} \int \frac{d \omega}{2 \pi}\left|G_{D}(\omega)\right|^{2}\left|G_{U}(\omega)\right|^{2}\left(f_{U}(\omega)-f_{D}(\omega)\right)$

$+|\lambda|^{2} \Gamma_{D} \Gamma_{U} \int \frac{d \omega}{2 \pi}\left|G_{D}\left(\omega+\frac{E_{R}-E_{L}}{2}\right)\right|^{2}\left|G_{U}\left(\omega-\frac{E_{R}-E_{L}}{2}\right)\right|^{2}$

$\times\left[\left\langle n_{R}\right\rangle\left(1-\left\langle n_{L}\right\rangle\right) f_{U}\left(\omega-\frac{E_{R}-E_{L}}{2}\right)\right.$

$\left.-\left\langle n_{L}\right\rangle\left(1-\left\langle n_{R}\right\rangle\right) f_{D}\left(\omega+\frac{E_{R}-E_{L}}{2}\right)\right]$

The positive direction is chosen to be from the higher chemical potential to the lower chemical potential, both for the electrons and protons.

3 Results and discussion The self-consistent equations for the electron and proton populations (e.g., as in Eq. (3)) were solved numerically and their solutions

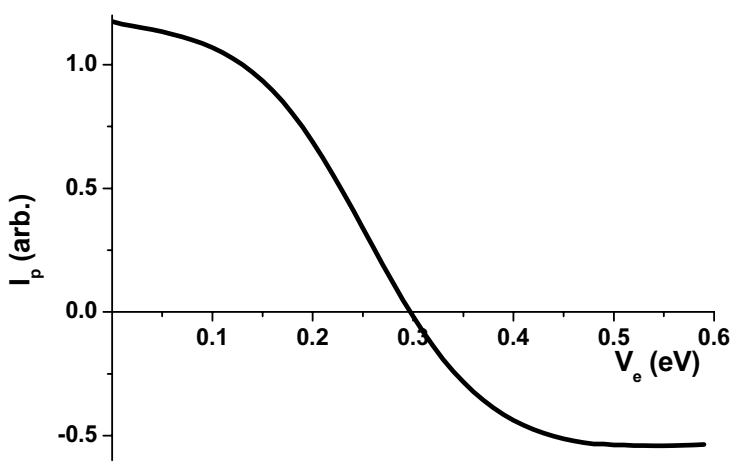

Figure 2 Proton current, $I_{p}$, as a function of electron voltage build-up. were substituted into Eqs. (7),(8) for $I_{e}$ and $I_{p}$. The electron current-voltage characteristics (not shown) exhibit the usual Ohmic region at small voltages, followed by a saturation regime.

The dependence of the proton current, $I_{p}$, on the electron voltage is shown in Fig. 2 for the electron energies $E_{R}$ $=100 \mathrm{meV}$ and $E_{L}=200 \mathrm{meV}$, proton energies $E_{U}=200$ $\mathrm{meV}$ and $E_{D}=100 \mathrm{meV}$ (the equilibrium chemical potentials are taken as the reference energies), tunnel splittings $\Delta_{a}=\Delta_{b}=10 \mathrm{meV}$, Förster constant, $=40 \mathrm{meV}$, and the temperature of the human body, $36.6^{\circ} \mathrm{C}$. The proton voltage build-up is taken to be $V_{p}=200 \mathrm{meV}$, which is typical for a living cell [1]. It is evident from Fig. 2 that at small electron voltages the proton current is positive and it becomes negative with increasing electron voltage. It should be noted that the negative proton current saturates at electron voltages larger than $450 \mathrm{meV}$, which is close to the maximal redox potential change along the $\mathrm{CcO}$ [1].

The temperature dependence of the proton current is of special interest. It is presented in Fig. 3 for the parameters of Fig. 2 and for the electron voltage $V_{e}=350 \mathrm{meV}$ corresponding to the linear regime of Fig. 2.

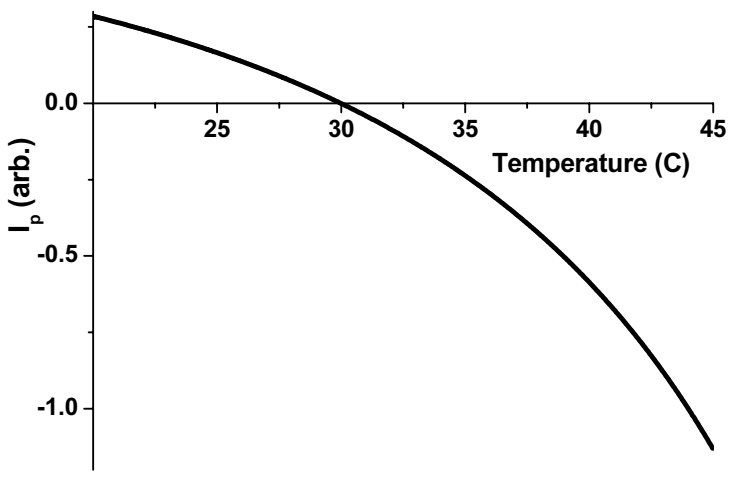

Figure 3 Temperature dependence of the proton current.

One can see that at temperatures below $30^{\circ} \mathrm{C}$, the proton current is positive and the proton pump cannot function. The optimal regime occurs at temperatures about that of the human body $\left(36^{\circ} \mathrm{C}\right)$, and with further temperature increase the negative proton current becomes so strong that it could break the electrostatic balance within the cell.

Förster-type processes require a resonant condition and are thus sensitive to detuning from the resonance. To illustrate this, we plot the proton (Fig. 4(a)) and electron (Fig. 4(b)) currents as functions of the detuning for varying $E_{U}$.

These figures show that the proton pump can only work if such detuning is sufficiently small. It should be noted that the electron current is strongly enhanced at the resonant condition because the energy drain provided by 

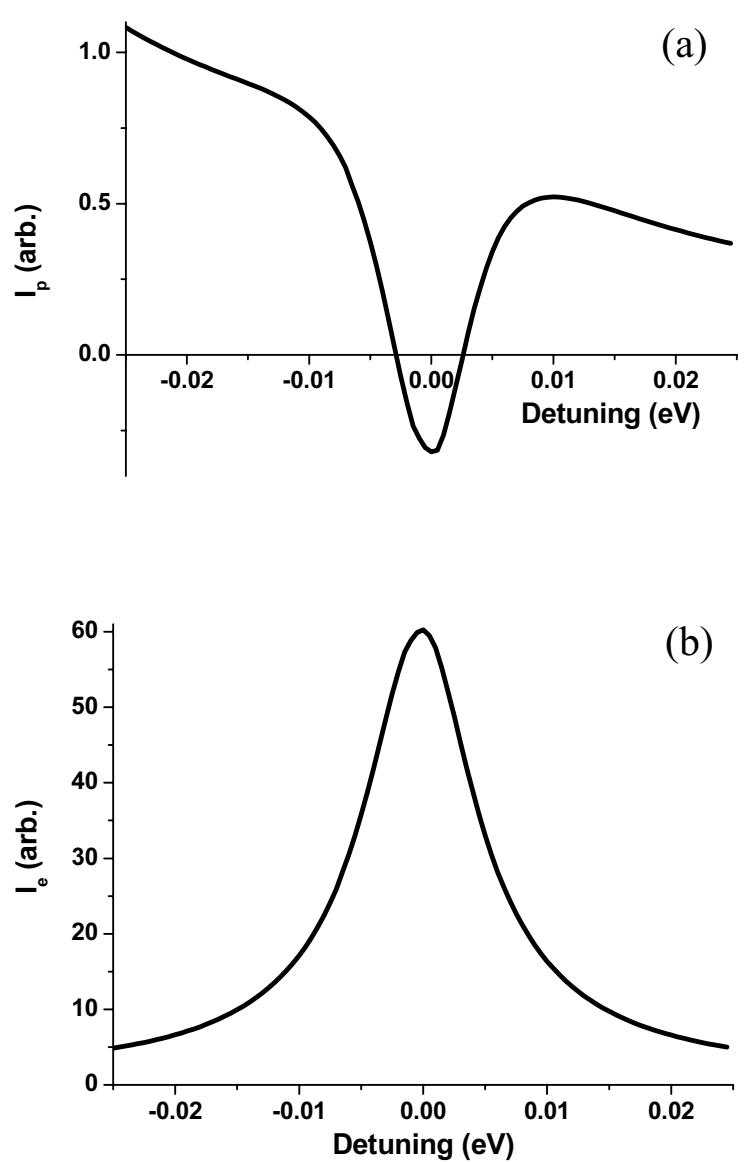

Figure 4 The proton, $I_{p}$, (a) and electron, $I_{e}$, (b) currents as functions of the detuning of the proton and electron energies from the resonant conditions.

the proton subsystem compensates the energy difference between electron levels in the two dots. The slope in Fig. 4(a) around the resonant current dip is produced by the direct proton current (the first term in Eq. (8)), which is maximal when $E_{U}=E_{D}$ and, therefore, increases when $E U$ decreases.

4 Conclusion In conclusion, we have proposed a model to describe the electronically-driven proton pump using a Förster-type resonant process for the energy transfer from the electron to proton subsystems. Our calculations, based on reasonable parameters, demonstrate that the proton pump works optimally at physiological temperatures. We have examined the effects of the electron voltage build-up, external temperature, and molecular electrostatics driving the electron and proton energies to the resonant conditions, and have shown that these parameters can control the proton pump operation.
Acknowledgements This work was supported in part by the National Security Agency, Army Research Office, Laboratory of Physical Sciences, and National Science Foundation grant No. EIA-0130383. The work of L.G.M. is partially supported by NSF NIRT, grant ECS-0609146.

\section{References}

[1] B. Alberts et al., Molecular Biology of the Cell (Garland Science, New York, 2002), Ch. 14.

[2] For recent reviews on proton pumps, see, e.g., J. P. Hosler, S. Ferguson-Miller, and D. A. Mills, Annu. Rev. Biochem. 75, 165 (2006); J. Quenneville, D. M. Popovic, and A. A. Stuchebrukhov, Biochem. Biophys. Acta 1757, 1035 (2006); M. Wikstrom and M. I. Verkhovsky, Biochem. Biophys. Acta 1757, 1047 (2006); S. Papa, G. Capitanio, and P. L. Martino, Biochem. Biophys. Acta 1757, 1133 (2006).

[3] T. Förster, in: Modern Quantum Chemistry, edited by O. Sinanogly (Academic, New York, 1965).

[4] See, e.g., A. Garg, J. N. Onuchic, and V. Ambegaokar, J. Chem. Phys. 83, 4491 (1985).

[5] M.-L. Tan, I. Balabin, and J. N. Onuchic, Biophys. J. 86, 1813 (2004).

[6] K. Faxen et al., Nature 437, 286 (2005).

[7] I. L. Medintz et al., Nature Mater. 2, 630 (2003).

[8] M. Achermann et al., Nature 429, 642 (2004).

[9] A. O. Govorov, Phys. Rev. B 71, 15532 